\title{
SMALL-SCALE HEATING EVENTS IN THE SOLAR ATMOSPHERE. I. IDENTIFICATION, SELECTION, AND IMPLICATIONS FOR CORONAL HEATING
}

\author{
N. Guerreiro ${ }^{1}$, M. Haberreiter ${ }^{1}$, V. Hansteen ${ }^{2}$, and W. Schmutz ${ }^{1}$ \\ ${ }^{1}$ Physikalisch-Meteorologische Observatorium Davos, World Radiation Center, Dorfstrasse 33, 7260 Davos, Switzerland; nuno.guerreiro@pmodwrc.ch \\ ${ }^{2}$ Institute of Theoretical Astrophysics, University of Oslo, P.O. Box 1029 Blindern, NO-0315 Oslo, Norway \\ Received 2015 June 5; accepted 2015 September 29; published 2015 October 28
}

\begin{abstract}
We present a comprehensive method to analyze small-scale heating events in detail in a 3D magnetohydrodynamics simulation for quiet-Sun conditions. The method determines the number, volume, and some general geometric properties of the small-scale heating events at different instants in a simulation with a volume of $16 \times 8 \times 16 \mathrm{Mm}^{3}$, spanning from the top of the convection zone to the corona. We found that there are about $10^{4}$ small-scale heating events at any instant above the simulated area of $128 \mathrm{Mm}^{2}$. They occur mainly at heights between 1.5 and $3.0 \mathrm{Mm}$. We determine the average value of their projected vertical extent, which ranges from 375 to $519 \mathrm{~km}$ over time, and we show that height, volume, and energy distribution of the events at any instant resemble power laws. Finally, we demonstrate that larger heating structures are a combination of much smaller heating events and that small-scale heating events dissipate enough energy to maintain the coronal energetic balance at any instant.
\end{abstract}

Key words: Sun: atmosphere - Sun: corona - Sun: flares - Sun: magnetic fields - Sun: transition region

\section{INTRODUCTION}

The fact that the solar corona is several orders of magnitude hotter than the photosphere is still an unresolved problem in astrophysics. This phenomenon has led to a multitude of theories aiming to explain the so-called coronal heating problem. It is generally accepted that overshooting convective motions have ample energy to sustain the corona. However, the mechanism responsible for the storage, transport, and release of the energy from the turbulent convection zone into the upper solar atmosphere is not yet fully understood. The magnetic field has been suggested as having an important role in the heating mechanism, but the exact contribution of the different magnetic processes such as dissipation of magnetohydrodynamic (MHD) waves and dissipation of magnetic energy in explaining the million-degree corona is not clear (Walsh \& Ireland 2003; Klimchuk 2006, 2015; Tomczyk et al. 2007; Reale 2010; van Ballegooijen et al. 2011; van Doorsselaere et al. 2011; Parnell \& De Moortel 2012; Goossens et al. 2013).

Generally, the sudden release of energy, known as flare-like events, which are associated with dissipation and reconnection of the magnetic field, has been observed and studied at different wavelengths (e.g., hard X-rays, soft X-rays, EUV, radio) on the Sun and other stars (Schaefer 1989; Haisch et al. 1991; Schaefer et al. 2000; Fletcher et al. 2011; Schrijver et al. 2012). Their energy is quasi-continuously distributed and spans from below the current observational detection limit, currently at about $\sim 10^{24}$ erg (Benz 2008; Aschwanden et al. 2014), up to $10^{35}$ erg or larger (Maehara et al. 2012).

As shown by Datlowe et al. (1974), Lin et al. (1984), Dennis (1985), and Crosby et al. (1993), the energy distribution of observed flare-like events follows a power law of the form

$$
\frac{d N}{d E} \propto E^{-\alpha},
$$

where $E$ is the flare energy and $d N$ is the number of events recorded within the energy interval $[E, E+d E]$. Hudson (1991) established the theory that if $\alpha<2$, the coronal heating is dominated by large events, whereas if $\alpha>2$, the events below the detectability limits dominate the heating instead.

Numerous observational studies of flare-like events with energies spanning several orders of magnitude have given different $\alpha$-values for similar and different energy ranges. Large-scale flares (superflares) on solar-like stars have an energy frequency distribution with $\alpha$ ranging from 2.0 to 2.3 (Maehara et al. 2012). Flares on the Sun show a distribution with $\alpha$ ranging from 1.44 to 2.5 (Yashiro et al. 2006; Aschwanden et al. 2014), while small-scale events give values of $\alpha$ from 1.3 to 2.6 (Aschwanden et al. 2014).

If a theory is to explain coronal heating based on flare-like events, it has to be able to explain the rather uniform heating supply needed to maintain the corona through the different stages of the solar cycle. Superflares have a very low occurrence rate on the Sun. Thus, they are not suitable candidates. In the case of flares, it is clear that in spite of their power, the energy supplied by them above active regions is not enough to maintain the corona over time. On the other hand, observational studies of small-scale heating events have led to contradictory results concerning their role in heating the corona. Therefore, they remain potential candidates to explain coronal heating.

Krucker \& Benz (1998) and Parnell \& Jupp (2000) used data from the Extreame-Ultraviolet Imaging Telescope (EIT; Delaboudinière et al. 1995) and the Transition Region and Coronal Explorer (TRACE; Handy et al. 1999), respectively, to study small-scale events in the solar atmosphere. They analyzed the peaks of the differential emission measure over time in order to identify the small-scale heating events, and they obtained a power law with $\alpha>2$. Those results support the idea that small-scale heating events can satisfy the energy requirements to explain coronal temperatures. On the other hand, Berghmans et al. (1998) and Aschwanden et al. (2000a, 2000b), also using EIT and TRACE data, respectively, found values of $\alpha<2$ for the same energy range. Their results suggest that small-scale heating events cannot account for the coronal heating. The discrepancy in the values of $\alpha$ from different analyses for the 
small-scale events stems primarily from the choice of the data sets and the methodology used to interpret them. For example, Krucker \& Benz (1998) and Parnell \& Jupp (2000) used a simple method based on the standard deviation to select the small-scale heating events. A local maximum is identified in the time line of a pixel, and the preceding local minimum or the start of the series is regarded as the background. Aschwanden et al. (2000a) used a more rigid selection criterion. They used the absolute maximum in the time line of a pixel and the absolute minimum as the background. In this case, co-spatial small-scale heating events were excluded and the methodology is biased against small-scale heating events (Benz \& Krucker 2002). In fact, the critical choices to be made when searching, identifying, and analyzing small-scale events are (1) which observational passband and temporal and spatial resolution should be used, (2) which event detection algorithms should be applied to the data sets, (3) how can the contribution of each event to the coronal heating be computed, and (4) how can the power law of the energy distribution be computed (e.g., Benz \& Krucker 2002). Clearly, these choices lead to systematic differences in determining $\alpha$.

An alternative method to assess the role of the small-scale heating events in coronal heating without having to calculate the exponent $\alpha$ is by calculating the energy flux into the atmosphere and determining whether it is sufficient to balance the coronal losses. The minimum energy flux required to maintain the coronal energetic balance in quiet-Sun conditions is estimated to be $3 \times 10^{5} \mathrm{erg} \mathrm{cm}^{-2} \mathrm{~s}^{-1}$ (Withbroe \& Noyes 1977) or higher, $4.6 \times 10^{5} \mathrm{erg} \mathrm{cm}^{-2} \mathrm{~s}^{-1}$, according to Krucker \& Benz (1998). Nevertheless, flux estimations based on events near the detectability limit have accounted only for a small fraction of the energy flux needed to maintain the corona (Krucker \& Benz 1998). Berghmans (2002) suggests that if the corona is to be heated by small-scale heating events, energies as low as $10^{18}$ erg need to be considered. This is several orders of magnitude below the current detectability limit.

Despite the challenges and ambiguities concerning smallscale events in recent studies, the suggestion that they have an important role in coronal heating has support from both observations and numerical simulations. The combination of observations from the Interface Region Imaging Spectrograph (IRIS; De Pontieu et al. 2014) instrument, coupled with the Atmospheric Imaging Assembly (AIA; Lemen et al. 2012) instrument on board the Solar Dynamics Observatory (SDO; Pesnell et al. 2012) spacecraft, has shown that the nonthermal electrons accelerated by small heating events deposit significant fractions of their energy in the chromosphere and transition region (Testa et al. 2014). Alternatively, numerical simulations of the braiding process of the magnetic field (Galsgaard \& Nordlund 1996; Hendrix et al. 1996; Browning et al. 2008; Bowness et al. 2013) and from 3D MHD models of the convection-corona system (Gudiksen \& Nordlund 2005; Hansteen et al. 2007; Bingert $\&$ Peter 2011) show that the small-scale heating scenario is able to generate a million-degree hot corona, which is maintained for extended periods of time with realistic values of the synthetic observable parameters (Peter et al. 2006; Hansteen et al. 2010). One of the advantages of these simulations is that processes below the detectability limit can be studied. However, the study of individual small-scale events with the aid of simulations is not trivial. One of the reasons is that the events are difficult to isolate because they overlap in time and their intermittence is not sufficiently high for them to stand out. Here, we build up on the Hansteen et al. (2015) work and present a newly developed method to at least partially overcome some of the challenges mentioned above. The method allows us to compute the properties of the different regions where energy dissipation occurs at any instant and helps us to shed further light on the role of small-scale heating events in coronal heating. Furthermore, an understanding of the physics responsible for the coronal heating might have farreaching implications such as improving the modeling capabilities of the variation of the coronal EUV emission (Haberreiter 2011; Haberreiter et al. 2014).

The paper is organized as follows: a short description of the MHD code and model used in this study is given in Section 2. The event definition, identification, and selection process and some of its applications are discussed in Section 3. Then, the implications for coronal heating of our results are presented in Section 4. Conclusions are given in Section 5.

\section{MODEL}

The simulated data for the analysis were produced with the Bifrost code (Gudiksen et al. 2011), and a detailed description is given by Hansteen et al. (2010) and Guerreiro et al. (2013). In summary, the code solves the time-dependent 3D MHD equations for a model that spans from the upper convection zone to the corona. The simulation includes non-gray radiative transfer with scattering for the photosphere and lower chromosphere (Hayek et al. 2010), an approximation to the non-LTE radiative terms that dominate the upper chromosphere (Carlsson \& Leenaarts 2012), and optically thin radiative losses in the transition region and corona. Thermal conduction along the magnetic field is also included and is dominant in the energy equation in the transition region and corona. The code has periodic boundary conditions in the $x$ - and $y$-directions and is nonperiodic in the $z$-direction. The lower boundary allows the flows to exit unimpeded, and the entropy is set to maintain the effective temperature close to the solar $T_{\text {eff }}=5780 \mathrm{~K}$ in the photosphere. The upper boundary has transparent boundary conditions. For a detailed description of the implementation of these terms refer to Gudiksen et al. (2011).

The computational box has a grid of $256 \times 128 \times 160$ points, which is equivalent to a volume in the Sun of $16 \times 8 \times 16 \mathrm{Mm}^{3}$. The vertical extent of the simulation box reaches from $2 \mathrm{Mm}$ below the photosphere to $14 \mathrm{Mm}$ above the photosphere into the corona. The zero point of the vertical scale is adopted to be where the monochromatic optical depth at $500 \mathrm{~nm}\left(\tau_{500}\right)$ is equal to 1 . For the $x$ - and $y$-directions a uniform grid of $65 \mathrm{~km}$ is chosen, while for the $z$-direction a nonuniform grid was used in order to ensure adequate resolution of the different atmospheric layers. The vertical grid spacing in the photosphere is $\Delta z=32 \mathrm{~km}, \Delta z=45 \mathrm{~km}$ at about $2 \mathrm{Mm}$ (transition region), and increases to $\Delta z=441 \mathrm{~km}$ above $10 \mathrm{Mm}$ in the corona. This model run for $1 \mathrm{hr}$ solar time and our use of the term snapshot represents the state of the simulation at varying instants, with a 10 s cadence.

\subsection{Heating in the Model}

In the atmospheric model used here the radiative losses in the lower atmosphere are balanced by an inflowing heat flux from the bottom boundary. The motions at the top of the convection zone and photosphere create acoustic waves that propagate into 
the chromosphere where the waves steepen and form shocks, which energize the lower layers of chromosphere. In the upper chromosphere and above, some $1000 \mathrm{~km}$ above the photosphere, where plasma- $\beta<1$, Joule dissipation is the main process responsible for the heating as the energy transferred to the magnetic field by the convective motions is dissipated (Gudiksen \& Nordlund 2005; Hansteen \& Gudiksen 2005; Hansteen et al. 2007; Bingert \& Peter 2011).

In this paper we are particularly interested in Joule dissipation, which acts in the resistive energy equation as a source term:

$$
\frac{\partial e}{\partial t}+\nabla(e \boldsymbol{u})+\boldsymbol{u} \nabla P=\nabla F_{\mathrm{r}}+\nabla F_{\mathrm{c}}+Q_{\text {Joule }}+Q_{\text {visc }},
$$

where $\boldsymbol{u}$ is the fluid velocity vector, $P$ is the gas pressure, $e$ is the internal energy, and $t$ is time. The term $F_{\mathrm{r}}$ stands for the radiative flux, $F_{\mathrm{c}}$ represents the conductive flux, and $Q_{\mathrm{Joule}}$ and $Q_{\text {visc }}$ are the Joule heating and viscous heating, respectively. $Q_{\text {Joule }}$ is responsible for converting magnetic energy into heat, and it is given by

$$
\begin{gathered}
Q_{\text {Joule }}=\eta j^{2}, \\
\boldsymbol{j}=\nabla \times \boldsymbol{B}, \\
\eta=\Delta d\left(\nu_{1} c_{f}+\nu_{2}|\boldsymbol{u}|+\nu_{3} \Delta d\left|-\nabla \cdot \boldsymbol{u}_{\perp}\right|_{+}\right),
\end{gathered}
$$

where $\eta$ is the resistivity in the model, $\boldsymbol{j}$ is the current density vector, $\boldsymbol{B}$ is the magnetic field vector, $\Delta d$ is the mesh size, and $\nu_{1}=0.02, \nu_{2}=0.3$, and $\nu_{3}=0.2$ are dimensionless coefficients that provide a suitable amount of dissipation of fast-mode waves $\left(\nu_{1}\right)$, advective motions $\left(\nu_{2}\right)$, and shocks $\left(\nu_{3}\right)$. Parameter $c_{f}$ is the fast-mode speed defined by $c_{f}=\sqrt{\left(B^{2}+\gamma p\right) / \rho}$, where $\gamma$ is the ratio of the specific heating rates, $\rho$ is the density, and $p$ is the pressure. Finally, $\left|-\nabla \cdot \boldsymbol{u}_{\perp}\right|_{+}$is the positive part of the compression rate $-\nabla \cdot \boldsymbol{u}$ that, when scaled with $\nu_{3} \Delta d$, prevents electrical current sheets from becoming numerically unsolvable.

The energy equation is solved using the numerical methods described in Gudiksen et al. (2011).

In summary, the average temperature structure of the model is maintained by the convective motion, radiative transfer in the photosphere, sound waves in the lower atmosphere, and Joule heating, thermal conduction, and radiative losses in the upper atmosphere (i.e., upper chromosphere, transition region, and corona). A comprehensive description of the atmospheric stratification in this model can be found in Hansteen et al. (2010), Guerreiro et al. (2013), and Hansteen et al. (2015).

\section{METHODOLOGY DEVELOPMENT: APPLICATIONS}

We introduce a method to identify, select, and study some of the properties of small-scale heating events in 3D MHD simulations at a particular instant in time. We use the terminology small-scale heating event to refer to the state of a small-scale Joule heating dissipation event at a certain instant. The term small-scale heating event does not refer to the total energy dissipated by a small-scale Joule heating event over its lifetime. Figure 1 is a 2D illustration of a small-scale heating event to aid the clarification of the definitions we used to implement the method and the parameters we intend to study.

The development of the method requires three a priori definitions: (1) the definition of a small-scale event, (2) the

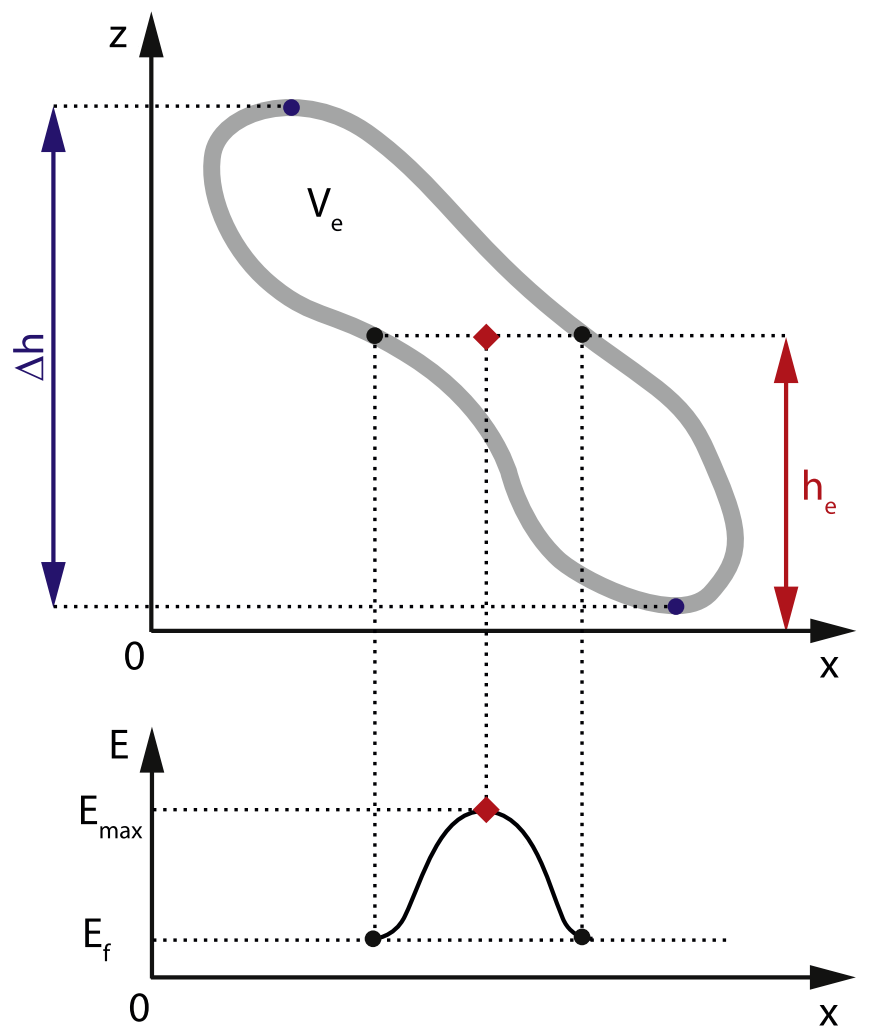

Figure 1. Illustration of a small-scale heating event and its boundary (thick gray line). The graph indicates the local maximum $E_{\max }$ (red diamond) and its corresponding threshold value $E_{f}=f E_{\max }$ (horizontal dashed line in the lower panel). The geometric parameters, small-scale heating event height $\left(h_{\mathrm{e}}\right.$, red arrow), projected vertical extent $\left(\Delta h\right.$, blue arrow), and volume $\left(\mathrm{V}_{e}\right)$ are also shown.

definition of a background, and (3) the definition of a threshold for Joule heating per unit mass $\left(Q_{\text {Joule }} / \rho\right)$, or equivalently per particle, to determine the volume, $\left(V_{e}\right)$, and energy, $\left(E_{e}\right)$, of the small-scale heating events. We define a small-scale heating event as a local maximum $\left(E_{\max }\right.$, red diamond in Figure 1) in the Joule heating per unit mass at any instant. This is based on the principle that a small-scale heating event at any stage of its lifetime always has a location inside its volume, where the Joule heating per unit mass is maximum for that specific smallscale heating event. Therefore, by locating the local maximum of the Joule heating per unit mass in the smallest possible region, we identify the individual small-scale heating events. Moreover, we use the ratio of Joule heating per unit mass to identify the local maxima because a high ratio presupposes a higher thermal energy $\left(E_{\mathrm{th}}\right)$. A location, i.e., grid cell, is accepted as the site of an event at a certain instant if and only if all 26 surrounding cells have a smaller Joule heating per unit mass. The baseline of the 27 cells used in the calculation of the maxima assures the best resolution of the small-scale heating events for the resolution of the simulation we used here. In addition, we consider the background to be the value below which we disregard all the local maxima. The background at any instant in time is considered to be all the cells with an energy dissipation rate below $10^{18} \mathrm{erg} \mathrm{s}^{-1}$. This choice of background is based on previous studies by Krucker \& Benz (1998), Parnell \& Jupp (2000), and Berghmans (2002) that if the heating events are to be relevant for coronal heating, we need to consider events with energy as low as $10^{18} \mathrm{erg}$ in order to satisfy the coronal energetic requirements. We assume the 
events to have a lifetime of at least $1 \mathrm{~s}$ and that their energy dissipation does not change significantly over a second, which is acceptable attending the results from Hansteen et al. (2015), where a study of the simulated events' lifetimes is presented, showing that they can live several minutes. Then, a rate of $10^{18} \mathrm{erg} \mathrm{s}^{-1}$ would therefore seem to be a reasonable choice for the background.

Finally, in order to determine the volume $\left(V_{e}\right.$; see Figure 1) of the small-scale heating events, we need an energy dissipation threshold $\left(E_{f}\right.$; see Figure 1$)$. The threshold is defined as a fixed percentage of every local maximum $\left(E_{f}=f E_{\max }\right)$. Since the local maxima are different, the energy threshold is different for the different small-scale heating events, even though the fixed percentage is the same. The volume of a small-scale heating event is composed of all the cells that have a path connectivity to the local maximum and have instantaneous energy dissipation above the energy dissipation threshold of the small-scale heating event. Notice that the volume of the small-scale heating events is not restricted to the 26 cells around the local maximum and it does not have to include all of the 26 cells either, as the cells are only included if they are above the energy threshold of the specific small-scale heating event. Additionally, we use a bottom-to-top approach to compute the instantaneous volumes of the small-scale heating events. This means that we order the local maxima from the lowest local maximum to the maximum local maximum for each snapshot and compute the volumes starting at the lowest local maximum. This prevents small-scale heating events with smaller local maxima from being incorporated into the volume of small-scale heating events with higher local maxima. Moreover, we do not restrict the small-scale heating events to have energy only above the background; only their local maxima are restricted to be so. However, the small-scale heating events, in general, are nearly only composed of cells with energy above the background because the volume of the first small-scale heating event calculated includes nearly all the background. Therefore, the volume of the first small-scale heating event is not considered in this work. Since, by construction, each grid cell can only be part of the volume of a single small-scale heating event for a certain snapshot, this implies that the volumes and energies of the small-scale heating events with local maxima close to the background are slightly underestimated.

The volume computation is challenging because we need to choose a threshold that gives the best representative results. If we use a conservative threshold, i.e., a high percentage of the local maximum, a large fraction of the energy of the individual small-scale heating events is excluded and the volume of the small-scale heating events is underestimated. On the other hand, if we choose a less restrictive threshold, the volumes computed may include cells where the energy is associated with different small-scale heating events. However, this is controlled by the bottom-up approach used in the method. Hence, this approach not only avoids small-scale heating events with different local maxima to be sampled in a single volume but also largely avoids the inclusion of cells in a smallscale heating event volume that belongs to a different smallscale heating event.

The threshold choice is especially critical for the small-scale heating events with maxima close to the background, because the local maxima have values very close to each other and the individual volumes of the small-scale heating events are harder to isolate. We tested several thresholds $(1 \%, 5 \%, 10 \%, 15 \%$, $20 \%$, and $25 \%$ ). As expected, the conservative thresholds $(15 \%, 20 \%$, and $25 \%)$ introduce a large underestimation of the total energy dissipated in each small-scale heating event at a certain instant. On the other hand, the less conservative thresholds $(1 \%, 5 \%, 10 \%)$, together with the bottom-up approach, give results with a smaller underestimation in the total energy dissipated by each individual small-scale heating event. Figure 2 displays the dissipation regions identified by the method at two instants $t=900 \mathrm{~s}$ (left) and $t=2800 \mathrm{~s}$ (right) using different thresholds $(1 \%, 5 \%, 10 \%)$. We first identified the local maxima, and then we computed the volumes of each small-scale heating event with a local maxima above the background and resampled the volumes of the small-scale heating events together in their original locations. As seen in Figure 2, the difference between the thresholds is small. Thus, we adopted the $1 \%$ threshold (i.e., $f=0.01$ ) in this study. We could have chosen a smaller threshold, but that would not have changed the results, since the cells that can be included in the volume of the small-scale heating events are constrained by (1) the choice of background, (2) the exclusion of the cells included in the volume of the first small-scale heating event calculated by the application of the bottom-up approach, and (3) the exclusion of the cells included in the volume of the small-scale heating events subsequently calculated by this approach. Therefore, there is no need to choose a threshold smaller than $1 \%$.

Notice that the number of grid points on the $z$-axis is less than in the full model, as described in Section 2. Since we only applied the method to the atmosphere and excluded the upper convection zone, only the atmosphere is displayed in the panels of Figure 2.

Figure 3 displays the total number of small-scale heating events $\left(N_{\mathrm{t}}\right.$, solid line) and the total energy dissipated into the modeled atmosphere ( $E_{\mathrm{t}}$, dashed line) by the small-scale heating events over time. The total number of small-scale heating events at each instant is given by the number of local maxima of the Joule heating per unit mass that are above the background, and the total energy released into the atmosphere by the small-scale heating events at each instant is obtained from adding the energy dissipated by all the small-scale heating events that have a local maxima above the background. The total number of small-scale heating events in each snapshot $\left(N_{\mathrm{t}}\right)$, increases over the first $200 \mathrm{~s}$ of the simulation. This rapid, indeed exponential, growth results from the collapse of magnetic gradients into current sheets at the beginning of the simulation, as explained by Galsgaard \& Nordlund (1996). Thereafter, the number of small-scale heating events ranges roughly from $9 \times 10^{3}$ to $1.2 \times 10^{4}$ per snapshot. The simulation box corresponds to a $16 \times 8 \mathrm{Mm}^{2}$ field of view on the Sun. If we assume the small-scale heating event occurrence rate to be homogeneously distributed over the whole solar atmosphere, we determine about $5 \times 10^{8}$ smallscale heating events at any instant. We note that this value may depend on the magnetic configuration of the model. As shown by Hansteen et al. (2015), the small-scale heating events in this model have lifetimes above 1 minute for a temporal resolution of $10 \mathrm{~s}$ in the simulation. This implies that when our approach is applied to consecutive snapshots with a cadence of $10 \mathrm{~s}$, the same small-scale heating event is counted multiple times at different states of its evolution. The small-scale heating events are first counted at the beginning of their evolution, and then 

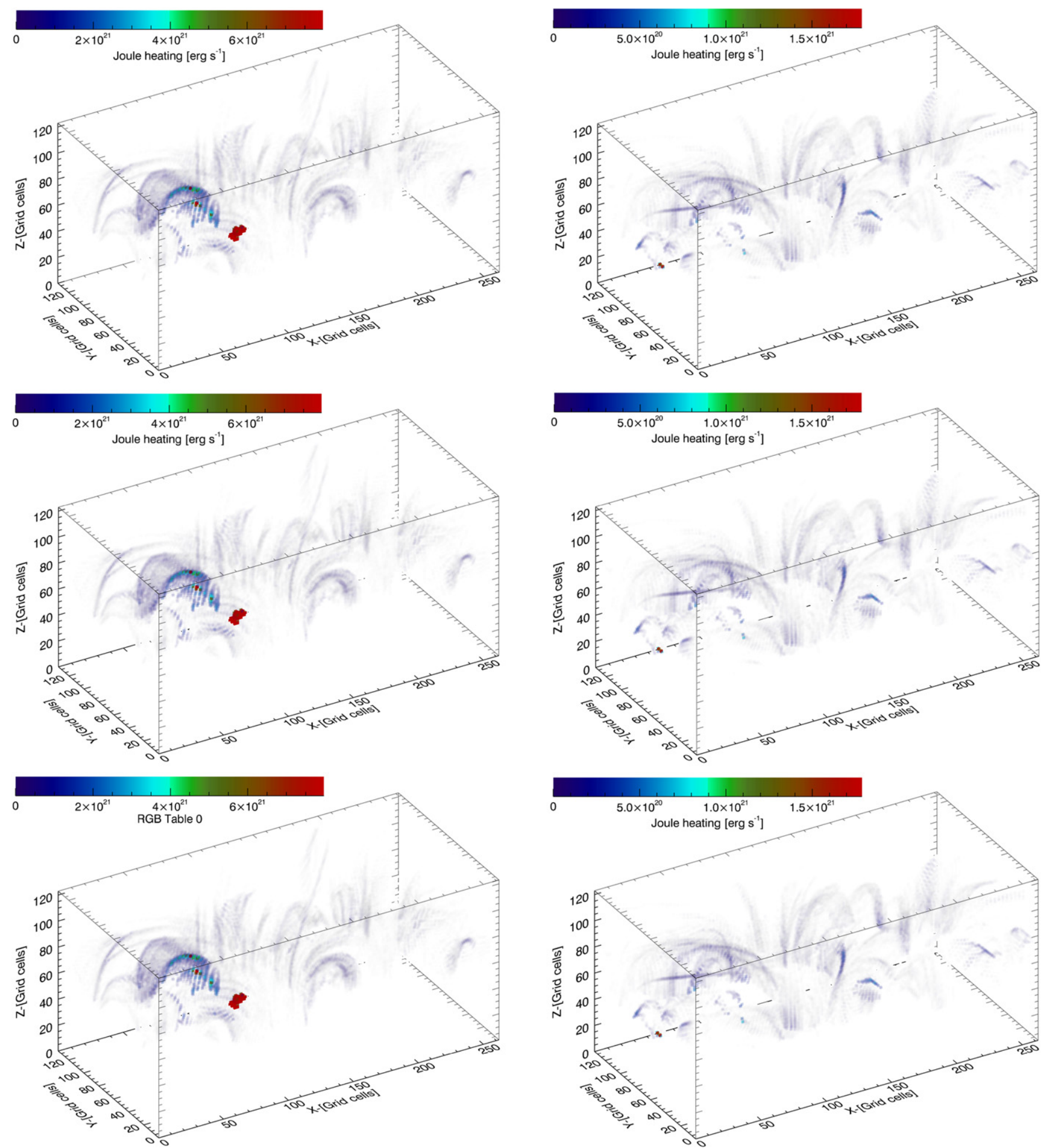

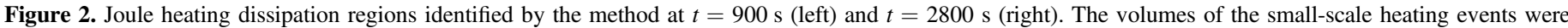

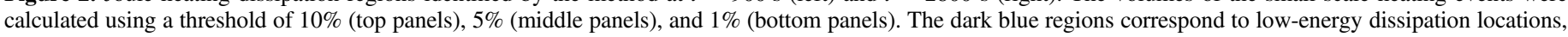
while red regions stand for high-energy dissipation. The transparent regions correspond to the background.

they evolve to release more and more energy per unit mass until they start decaying, ultimately vanishing below the background value. The other important aspect of Figure 3 is that it helps to understand that the large heating structures we see in Figure 2 are a combination of a large number of smaller small-scale heating events. For example, if we consider the snapshot at $t=2800 \mathrm{~s}$, we can see that there are about
$12 \times 10^{3}$ small-scale events that combine into the heating structures shown in the right panels of Figure 2. This supports previous conclusions that larger current sheets or large flarelike bursts of energy are a combination of small-scale heating events (Parker 1987).

The total energy dissipated $\left(E_{t}\right.$; shown in Figure 3$)$ by the small-scale heating events at any instant, with local maxima 


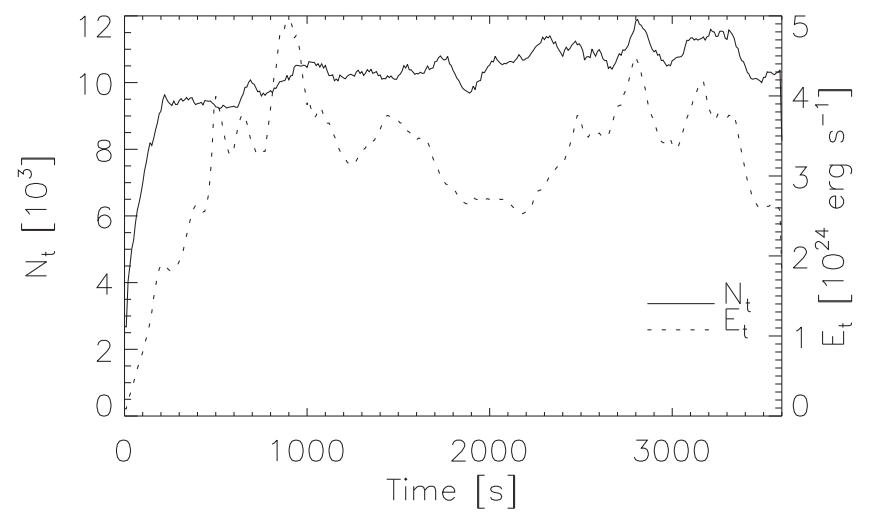

Figure 3. Total number of small-scale heating events identified in the simulation $\left(N_{\mathrm{t}}\right.$, solid line), and total energy dissipated $\left(E_{\mathrm{t}}\right.$, dashed line) by the computed small-scale heating events for each snapshot.

above the background, shows that a smaller amount of energy is released into the atmosphere at the beginning of the simulation, but it rapidly increases in the first $500 \mathrm{~s}$. There are seven major energy peaks of $E_{\mathrm{t}}$ : at about $400,600,900,1400,2450,2800,3150 \mathrm{~s}$, where a total energy rate of $4.0 \times 10^{24}, 3.8 \times 10^{24}, 5.0 \times 10^{24}, 3.8 \times 10^{24}$, $3.7 \times 10^{24}, 4.4 \times 10^{24}, 4.2 \times 10^{24} \mathrm{erg} \mathrm{s}^{-1}$ is released by the small-scale heating events, respectively. Generally, the dotted line shows a release of energy into the atmosphere of the order of $10^{24} \mathrm{erg} \mathrm{s}^{-1}$. This energy corresponds to about $92 \%$ of the energy dissipated by all the detected small-scale heating events, which includes those with local maxima above and below the background.

The peaks of $E_{\mathrm{t}}$ and the peaks of $N_{\mathrm{t}}$ are sometimes correlated, for example, from $t=2700 \mathrm{~s}$ to $t=3000 \mathrm{~s}$ or anticorrelated from $t=800 \mathrm{~s}$ to $t=1000 \mathrm{~s}$. The peaks of energy dissipation from $t=800 \mathrm{~s}$ to $t=1000 \mathrm{~s}$ result from small-scale heating events that dissipate larger amounts of energy per second, since the number of events does not change significantly. This is shown in the left panels of Figure 2, which display the simulation at $t=900 \mathrm{~s}$. The panels show that an anticorrelation between the number of small-scale heating events and the energy dissipated is due to a large number of small-scale heating events that dissipate a larger amount of energy. On the other hand, toward the end of the simulation the energy dissipation peaks seem to be correlated with an increase in the number of small-scale heating events. As can be seen in the right panels of Figure 2, which shows the state of the simulation at $2800 \mathrm{~s}$, the number of small-scale heating events dissipating higher amounts of energy is small. Therefore, the increase in the total energy dissipation is a consequence of the increase of the number of small-scale heating events.

We apply our method to study several parameters of the small-scale heating events, i.e., height $\left(h_{e}\right.$, red arrow in Figure 1), projected vertical extent $(\Delta h$, blue arrow in Figure 1), volume $\left(V_{e}\right)$, and energy $\left(E_{e}\right)$ for each snapshot.

The $h_{e}$ of a small-scale heating event is the distance from the photosphere $(z=0)$ to the location of its local maximum, i.e., this parameter gives the heights in the atmosphere where the small-scale heating events occur. The parameter $\Delta h$ refers to the distance from the lowest to the highest edge of the smallscale heating event along the $z$-axis; it is represented by the blue arrow in Figure 1 . The $\mathrm{V}_{e}$ corresponds to all the cells with a path connectivity that obey the equation given by $E_{f}=f E_{\max }$, where $E_{\max }=Q_{\text {Joule }} / \rho$ is the local maximum
Joule heating per unit mass of a small-scale heating event, and $f=0.01$ is the $1 \%$ corresponding to the threshold we chose. In the case of Figure 1, it corresponds to the area limited by the gray line. The $E_{e}$ is the integration of the Joule heating of each cell contained in the volume of the individual small-scale heating events.

Figure 4 displays the $V_{e}$ (left), $\Delta h$ (middle), and $E_{e}$ (right) distribution of the small-scale heating events at $t=900 \mathrm{~s}$ (top panels), $t=2000 \mathrm{~s}$ (middle panels), and $t=2800 \mathrm{~s}$ (bottom panels). These variables have distributions that resemble a power law at each instant.

The smallest possible volume for a small-scale heating event is one cell, which, depending on its location, can correspond to different volumes owing to the nonuniform grid in the $z$ direction. This situation happens if the 26 cells surrounding the local maximum have energy that is below the energy threshold of that small-scale heating event. The largest volume for the cases shown is roughly $7 \times 10^{9}(\mathrm{~km})^{3}$. Overall, the majority of the small-scale heating events have volumes smaller than $5 \times 10^{8}(\mathrm{~km})^{3}$.

The $\Delta h$ distributions show that the majority of the smallscale heating events have a height that is smaller than $3 \mathrm{Mm}$ for the instants represented. The smallest height comes from the small-scale heating events whose volume is composed of a single cell, and these are generally small-scale heating events with local maxima very close to the background. This suggests that such small-scale heating events are at the beginning or at the end of their lifetime. The values of the height range shown here represent the height range of the small-scale heating events at different stages of their evolution. The largest heights are the order of $3.5 \mathrm{Mm}$ for the cases displayed.

The energy distributions show that the small-scale heating events selected, at the displayed instants, have an energy dissipation ranging roughly from $10^{18}$ to $10^{23} \mathrm{erg} \mathrm{s}^{-1}$. We chose a bin size to be $1.0 \times 10^{18} \mathrm{erg} \mathrm{s}^{-1}$, which is similar to the background value. As mentioned above, the bulk of the data in the distribution resemble a power law. However, if the distribution of the small-scale heating events follows a power law, there is a shortfall of the small-scale heating events for lower energies, since a fit to the bulk of the data suggests a higher number of small-scale heating events than computed. This behavior has also been identified in observational data by Krucker \& Benz (1998) and Parnell \& Jupp (2000) when they studied the energy distribution of small-scale heating events. Parnell \& Jupp (2000) suggested that the shortfall of the smallscale heating events was a consequence of the low spatial resolution of TRACE. In our case, the shortfall of the smallscale heating events at low energies results from an underestimation of the energy of the small-scale heating events that have local maxima close to the background as explained above.

The thermal energy, $E_{\mathrm{th}}$, of observed flare-like events has been frequently used to estimate their energy distributions. It is given by $E_{\mathrm{th}}=3 k_{\mathrm{B}} n_{e} T_{e} V$, where $k_{\mathrm{B}}$ is the Boltzmann constant, $n_{e}$ is the electron density, $T_{e}$ is the flare peak temperature, and $V$ is the flare volume. However, the volume of the flare-like events cannot be directly measured. This has forced several authors to use different scaling laws to estimate it. Krucker \& Benz (1998) and Parnell \& Jupp (2000) estimated that the volume of the small-scale heating events was approximately given by $V=A h$, where $A$ is the observed flare area, which can be directly measured from the observations, and $h$ is the height of the small-scale heating event, which cannot be directly 

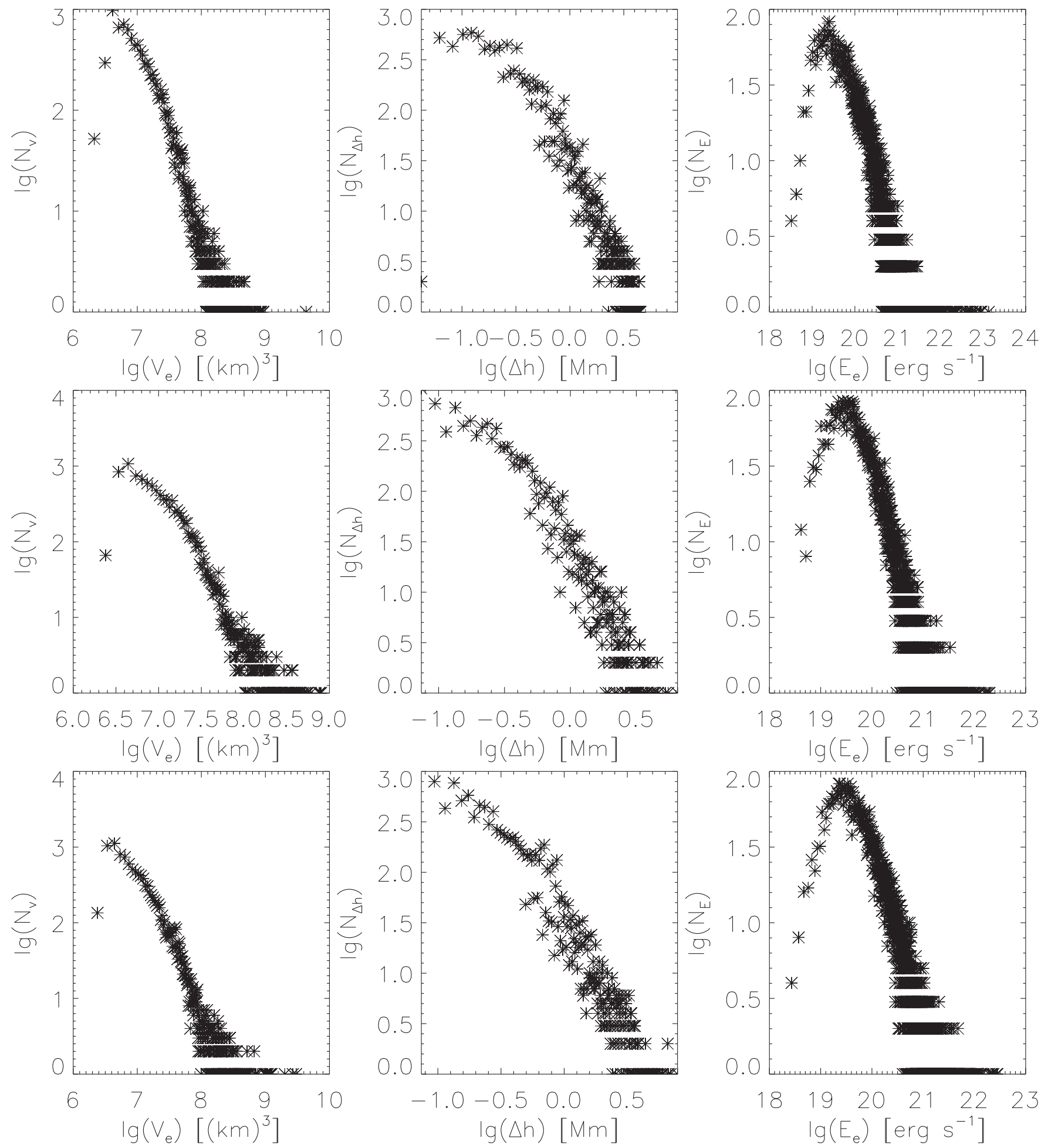

Figure 4. Number distribution of the volume $\left(V_{e}\right.$; left), projected vertical extent $\left(\Delta h\right.$; middle), and Joule heating $\left(E_{e}\right.$; right) of the small-scale heating events at $t=900 \mathrm{~s}$ (top panels), $t=2000 \mathrm{~s}$ (middle panels), and $t=2800 \mathrm{~s}$ (bottom panels).

measured. Krucker \& Benz (1998) assumed a constant value for $h$ of $5000 \mathrm{~km}$, while Parnell \& Jupp (2000) considered two options for $h$ : (1) a constant value for $h$ of $726 \mathrm{~km}$, which is equal to the width of a pixel, and (2) $h$ is proportional to $\sqrt{A}$.

The height values used by Krucker \& Benz (1998) and Parnell \& Jupp (2000) to calculate the $E_{\text {th }}$ can be compared to our results from the simulation.
Figure 5 displays the average $\Delta h(\langle\Delta h\rangle)$ for each snapshot over the full simulation run. Currently, we do not follow the individual small-scale heating events over their lifetime, so we cannot calculate the variation of the $\Delta h$ for the individual small-scale heating events over time. However, we calculate the $\Delta h$ of the different small-scale heating events at the individual snapshots, which gives the $\Delta h$ of the small-scale 


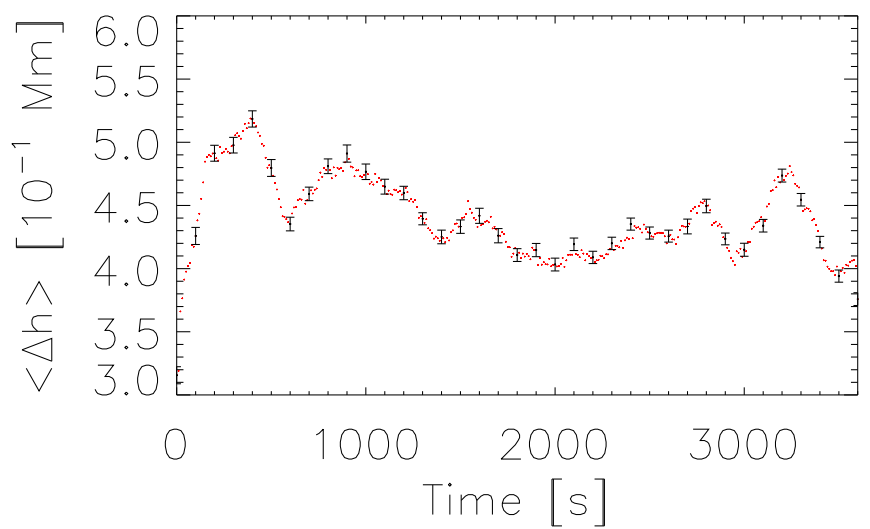

Figure 5. Average projected vertical extent of the small-scale heating events $(\langle\Delta h\rangle)$ over time. The error bars are only displayed at every 10th data point.

heating events at different stages of their evolution. Thus, it is reasonable to assume that $\langle\Delta h\rangle$ is a good estimate of the height of the small-scale heating events in general. The error bars were derived from a Monte Carlo simulation (Manno 1999) and represent the statistical uncertainty of the average. The value of $\langle\Delta h\rangle$ ranges from $375 \pm 5 \mathrm{~km}$ to $519 \pm 6 \mathrm{~km}$ after the simulation has relaxed. The values of $\langle\Delta h\rangle$ we obtained are much smaller than those used by Krucker \& Benz (1998) to calculate the thermal energy of the small-scale heating events. On the other hand, $\langle\Delta h\rangle$ is in agreement with the height of the small-scale heating events suggested by Benz \& Krucker (2002) and about $200 \mathrm{~km}$ smaller than the values assumed by Parnell \& Jupp (2000). This result also means that the thermal energies computed by Krucker \& Benz (1998) and Parnell \& Jupp (2000) are overestimated.

Figure 6 displays the probability distribution function (PDF) for $h_{e}$ (left) and $\Delta h$ (right) versus the energy of the small-scale heating events $\left(E_{e}\right)$ at $t=900 \mathrm{~s}$ (top panels), $t=2000 \mathrm{~s}$ (middle panels), and $t=2800 \mathrm{~s}$ (bottom panels). The left panels displaying the PDF for $h_{e}$ against $E_{e}$ show that the small-scale heating events typically occur above $1.5 \mathrm{Mm}$ and below $9 \mathrm{Mm}$ with the majority of them in the interval from 1.5 to $3.0 \mathrm{Mm}$. The number of small-scale heating events computed in the upper transition region and corona is small. In addition, the total energy dissipated by the small-scale heating events occurring in the upper transition region and corona is smaller than the total energy dissipated by the events in the lower layers. The more energetic small-scale heating events generally occur in the upper chromosphere and transition region, roughly from 1.5 to $4 \mathrm{Mm}$. Note that the temperature stratification in the model can be roughly described as a function of height by the following: photosphere from $z=0 \mathrm{Mm}$ to $z=0.7 \mathrm{Mm}$, chromosphere from $z=0.7 \mathrm{Mm}$ to $z=1.7 \mathrm{Mm}$, transition region from $z=1.7 \mathrm{Mm}$ to $z=4 \mathrm{Mm}$, and corona is above $z=4 \mathrm{Mm}$. This description of the atmosphere in the model is by no means complete and aims to illustrate the regions where the events happen. For a broad discussion on the atmosphere stratification in this model please refer to Hansteen et al. (2015). The panels displaying the PDF of the $\Delta h$ versus $E_{e}$ show that the majority of the small-scale heating events are smaller than $200 \mathrm{~km}$ at the instants presented here. In general, the larger the $\Delta h$ of a small-scale heating event, the more energetic it is, as shown in the top and bottom panels. The middle panel on the right, on the other hand, shows that smallscale heating events with a large $\Delta h$ can have low energy, but they seem to be less frequent. A general conclusion from this figure is that the majority of the small-scale heating events are releasing energy in the range $10^{19}$ to $10^{21} \mathrm{erg} \mathrm{s}^{-1}$ at each instant.

In summary, the advantages of this method are that it can help to circumvent some of the critical choices usually made when searching, identifying, and analyzing small-scale heating events. Since we use the maxima in the Joule heating per unit mass to identify the individual small-scale heating events, the identification is independent of the passband; therefore, both strong and weaker events are identified at once. Moreover, the use of a 27-cell baseline makes it possible to resolve different small-scale heating events that might be relatively close together that otherwise could be considered as the same small-scale heating event. At the same time, it is possible to attribute a minimum energy contribution from each small-scale heating event to the total energy ejected into the atmosphere at each instant. Therefore, we can estimate the amount of energy injected into the atmosphere at any instant and compare it with the energy required to maintain the energy balance of the corona. This implies that we can study the contribution of the small-scale heating events for the coronal heating without having to use a power law. This avoids all the ambiguities associated with the fitting of it.

\section{IMPLICATIONS FOR CORONAL HEATING}

The possibility to calculate the total energy dissipated at any instant using our method allows us to calculate the total flux of energy ejected into the corona. This value can be compared with the flux value required to maintain the coronal balance calculated by Withbroe \& Noyes (1977) and Krucker \& Benz (1998).

Withbroe \& Noyes (1977) calculated that the energy flux necessary to maintain the coronal energy balance is $3 \times 10^{5} \mathrm{erg} \mathrm{cm}^{-2} \mathrm{~s}^{-1}$ in quiet-Sun conditions, while Krucker \& Benz (1998) suggested a higher flux of $4.6 \times 10^{5} \mathrm{erg} \mathrm{cm}^{-2} \mathrm{~s}^{-1}$.

Figure 7 shows the energy flux $\left(F_{E}\right)$ into the atmosphere during $1 \mathrm{hr}$ of solar time, and it is given by $F_{E}=E_{t} / A_{s}$ at each instant, where $A_{s}$ is the area of the simulation at $z=0$. The different curves show the contribution of the small-scale heating events, with local maxima above the background that are above a certain energy scale, to the flux at each instant. The contribution of all the small-scale heating events with local maxima above $1 \times 10^{18} \mathrm{erg} \mathrm{s}^{-1}$ is given by the solid line. The dotted line represents small-scale heating events with instantaneous energy dissipation above $1 \times 10^{20} \mathrm{erg} \mathrm{s}^{-1}$, the dashed line represents small-scale heating events with energy dissipation greater than $1 \times 10^{21} \mathrm{erg} \mathrm{s}^{-1}$, and the dot-dashed line represents the small-scale heating events with an instantaneous energy dissipation greater than $1 \times 10^{22} \mathrm{erg} \mathrm{s}^{-1}$.

These curves show that for the model we are using, the total number of small-scale heating events that have an energy dissipation rate above $10^{21} \mathrm{erg} \mathrm{s}^{-1}$ (dashed line) at any instant is more than sufficient to provide enough energy flux to maintain the coronal balance according to Withbroe \& Noyes (1977) and Krucker \& Benz (1998). Therefore, this suggests that the smallscale heating events can provide enough energy to explain coronal heating. On the other hand, in this model the number of small-scale heating events with energy above $1 \times 10^{22} \mathrm{erg} \mathrm{s}^{-1}$ at any instant cannot fulfill the coronal energy requirements. These results are similar to the findings of Gudiksen \& Nordlund (2005), who demonstrated that the magnetic field 

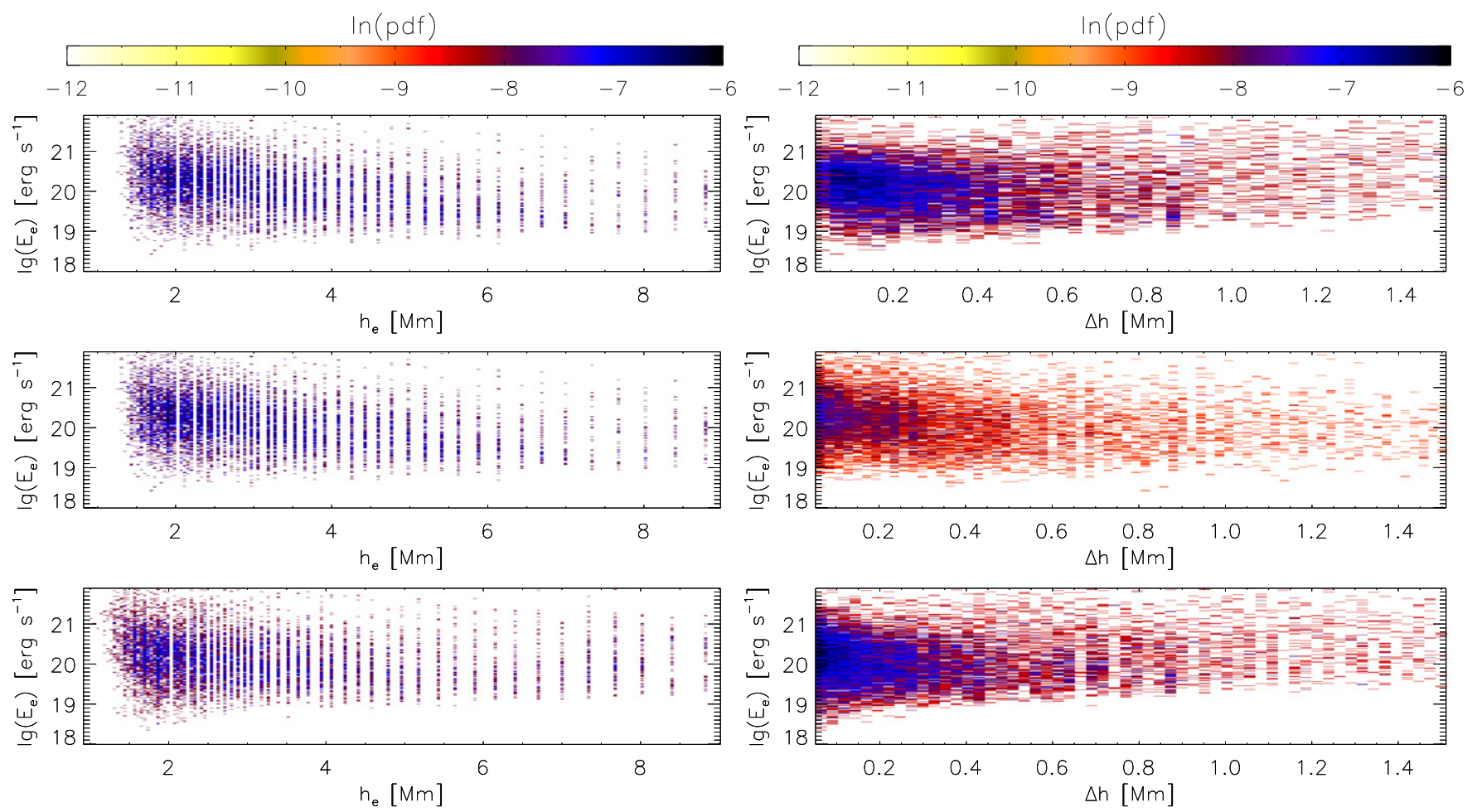

Figure 6. PDF of the energy $\left(E_{e}\right)$ vs. height $\left(h_{e} ;\right.$ left) and projected vertical extent ( $\Delta h$; right) at $t=900 \mathrm{~s}$ (top), $t=2000 \mathrm{~s}$ (middle), and $t=2800 \mathrm{~s}$ (bottom). The color ranges from white for a low probability of a small-scale heating event to black for a high probability of a small-scale heating event.

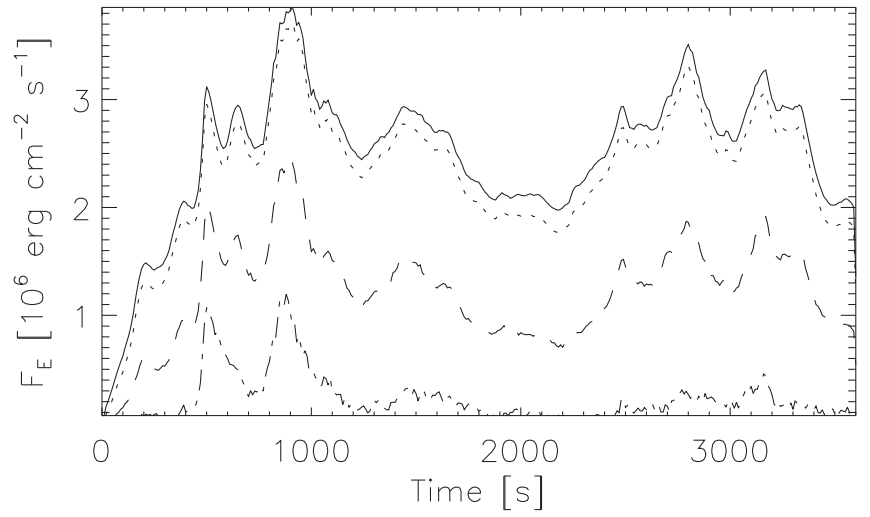

Figure 7. Energy flux into the atmosphere for each snapshot over time. The solid line represents the total energy flux computed considering all the smallscale heating events above the background. The dotted, dashed, and dot-dashed lines correspond to the total flux computed from small-scale heating events with an energy dissipation at any instant greater than $10^{20}, 10^{21}, 10^{22} \mathrm{erg} \mathrm{s}^{-1}$, respectively.

was able to dissipate enough energy in the case of an active region to maintain the coronal temperature at about $\sim 10^{6} \mathrm{~K}$. On the other hand, we focus on quiet-Sun conditions and demonstrate that the magnetic field can dissipate enough energy to maintain the quiet corona at temperatures on the order of a million degrees kelvin. Essentially, we relate the energy flux that maintains the corona at about $\sim 10^{6} \mathrm{~K}$ in quietSun conditions with the specific small-scale heating events at any instant.

The energy flux of all the small-scale heating events we detect (solid line in Figure 7) is about an order of magnitude greater than the heating requirement for the quiet corona as obtained by Withbroe \& Noyes (1977) and Krucker \& Benz (1998).

The difference between the energy flux obtained from the 3D MHD simulations and the observational data can have two reasons. First, the magnetic field topology and strength at the photosphere can influence the energy dissipated. However, measurements of the pointing flux for the model we used here give values inside the observed ranges (Hansteen et al. 2015). Second, the choice of parameters in the method we developed can also lead to the discrepancy of the energy flux. The first parameter that can have implications for the energy flux computed is the background. If we were to choose a lower background value, we would get a larger flux because more events would be included in the small-scale heating event sample used for the calculation, while a higher background value would give a smaller flux, since we would get a sample with less small-scale heating events.

The second parameter that could possibly influence the value of the total energy dissipated at each instant is the threshold $(f)$ chosen to determine the volume of the events. However, the construction of the method naturally limits the energy dissipated by each small-scale heating event at any instant to be greater than the background or $10^{18} \mathrm{erg} \mathrm{s}^{-1}$ rate. As described in the previous section, the volume of the first small-scale heating event calculated contains nearly all the background grid cells, therefore limiting the volume of the remaining small-scale heating events to include nearly only cells that have energy dissipation rates higher than the background rate. Thus, essentially the only choice with implication for the calculation of the energy flux is the background. Our choice of background is based on the assumption that if the small-scale heating events are to be 
responsible for the coronal heating, we need to consider smallscale heating events with energy as low as $10^{18} \mathrm{erg}$. If the choice of background is responsible for the excess of energy, then it is reasonable to assume that we do not have to consider events as low as $10^{18} \mathrm{erg}$ to satisfy the coronal energetic balance; a lower limit of about $10^{20} \mathrm{erg}$ seems more adequate. On the other hand, the energy flux estimated by Krucker \& Benz (1998) for quiet-Sun conditions is an underestimate because they only considered radiative losses to estimate the energy flux. Their estimate is larger than the value that Withbroe \& Noyes (1977) put forward for quiet-Sun conditions. This implies an underestimate of the energy flux computed by Withbroe \& Noyes (1977). Therefore, it is difficult at this stage to estimate the discrepancy of the energy flux results in relation to the flux computed by Krucker \& Benz (1998) and Withbroe \& Noyes (1977). It is then not clear how much of the flux difference is a direct consequence of the background choice. A detailed study of the energy balance in the locations of the small-scale heating events is needed to better understand the variations of the thermal energy resulting from the small-scale heating events and the discrepancy between the energy flux we compute and the energy flux given by observations.

\section{CONCLUSION}

A comprehensive method to identify, select, and analyze small-scale heating events in a 3D MHD simulation of the solar atmosphere was presented. Applying the method to the MHD simulation, which corresponds to an area on the solar surface of $128 \mathrm{Mm}^{2}$, we found about $10.5 \times 10^{3}$ small-scale heating events above the background per snapshot. This corresponds to about $5 \times 10^{8}$ small-scale heating events over the whole solar atmosphere at any instant for quiet-Sun conditions. The technique we apply to detect the small-scale heating events also shows that the large heating structures are a combination of a much larger number of small-scale heating events.

The average projected vertical extent, $\langle\Delta h\rangle$, of the smallscale heating events ranges from 375 to $519 \mathrm{~km}$. The value is smaller than the constant values used by Krucker \& Benz (1998) and Parnell \& Jupp (2000) to estimate the thermal energy, suggesting that their results are overestimated. A value of about $450 \mathrm{~km}$ is probably the most accurate value when estimating the thermal energy of small-scale heating events assuming a constant height as also suggested by Benz \& Krucker (2002). The majority of the small-scale heating events occur at approximately $2 \mathrm{Mm}$ from the photosphere, but their height of occurrence ranges from 1.3 to $9 \mathrm{Mm}$ over the whole simulation. This shows that even though the heating can occur in all layers from the chromosphere to the corona, the bulk of the heating can be attributed to small-scale heating events confined to cooler atmospheric layers. The volume $\left(V_{e}\right)$, projected vertical extent $(\Delta h)$, and instantaneous energy dissipation $\left(E_{e}\right)$ exhibit distributions similar to those of a power law. This is consistent with a scenario where the magnetic field in the upper layer of the solar atmosphere is in a self-organized critical state (Lu \& Hamilton 1991), where the power laws are a direct consequence of the physics of such a state (Bak et al. 1987, 1988).

The energy distribution shows that we compute small-scale heating events with an instantaneous energy dissipation ranging roughly from $1 \times 10^{18}$ to $2 \times 10^{23} \mathrm{erg} \mathrm{s}^{-1}$ at each instant.
We find an energy flux into the corona at any instant that is sufficient to maintain the coronal balance in a quiet-Sun scenario when considering only the small-scale heating events with an instantaneous energy dissipation greater than $1 \times 10^{21} \mathrm{erg} \mathrm{s}^{-1}$. This result suggests that we might only need to observe events down to $1 \times 10^{20} \mathrm{erg}$ in order to observationally verify the role of small-scale heating events for coronal heating instead of the $1 \times 10^{18} \mathrm{erg}$ as previously thought. If we consider all the events with an energy dissipation above $1 \times 10^{18} \mathrm{erg} \mathrm{s}^{-1}$, we compute a flux that is about an order of magnitude greater than the fluxes presented by Withbroe \& Noyes (1977) and Krucker \& Benz (1998). At this stage it is difficult to assess the fraction of the "excess" of energy flux that results from our choice of background, since it is not clear to what extent the energy fluxes are underestimated by Withbroe \& Noyes (1977) and Krucker \& Benz (1998). Nevertheless, our results support a scenario where the quiet corona is heated mainly by small-scale heating events.

Results in this study contribute to a better understanding of small-scale heating events in the solar atmosphere and are important for the potential identification of small-scale heating events by the upcoming observations from the Extreme Ultraviolet Imager (EUI; Halain et al. 2014) and the Spectral Imaging of the Coronal Environment (SPICE; Fludra et al. 2013).

In this paper we presented the overall approach and results of identifying and analyzing small-scale heating events in 3D MHD simulations at different instants of their evolution. Despite the new approach to analyzing small-scale heating events, we are limited by the fact that we only analyze one model with a rather low resolution. Further studies are being conducted in models with higher resolution and with different magnetic field configurations in the photosphere. The work in progress also includes the study of the time evolution of the small-scale heating events, their energy balance, and the response of optically thin spectral lines to the small-scale heating events.

N.G. is thankful for the financial support from the Swiss National Science Foundation through grant $n^{o} 153302$. He is also thankful to $\mathrm{S}$. Nyeki for relevant comments on this paper. The authors are thankful for the constructive comments from the referee. Furthermore, the research leading to these results has also benefited from the European Community, Seventh Framework Program (FP7 2012) under Grant Agreement $\mathrm{n}^{o}$ 313188 (SOLID, http://projects.pmodwrc.ch/solid/).

\section{REFERENCES}

Aschwanden, M. J., Crosby, N., Dimitropoulou, M., et al. 2014, SSRv, 1 Aschwanden, M. J., Tarbell, T., Nightingale, R., et al. 2000b, ApJ, 535, 1047 Aschwanden, M. J., Nightingale, R. W., Tarbell, T. D., \& Wolfson, C. J. 2000, ApJ, 535, 1027

Bak, P., Tang, C., \& Wiesenfeld, K. 1987, PhRvL, 59, 381

Bak, P., Tang, C., \& Wiesenfeld, K. 1988, PhRvA, 38, 364

Benz, A. O. 2008, LRSP, 5, 1

Benz, A. O., \& Krucker, S. 2002, ApJ, 568, 413

Berghmans, D. 2002, in ESA Special Publication 506, Solar Variability: From Core to Outer Frontiers, ed. A. Wilson (Noordwijk: ESA), 501

Berghmans, D., Clette, F., \& Moses, D. 1998, A\&A, 336, 1039

Bingert, S., \& Peter, H. 2011, A\&A, 530, A112

Bowness, R., Hood, A. W., \& Parnell, C. E. 2013, ApJ, 560, 89

Browning, P. K., Gerrard, C., Hood, A. W., Kevis, R., \& van der Linden, R. A. M. 2008, A\&A, 485, 837

Carlsson, M., \& Leenaarts, J. 2012, A\&A, 539, A39

Crosby, N. B., Aschwanden, M. J., \& Dennis, B. R. 1993, SoPh, 143, 275 
Datlowe, D. W., Elcan, M. J., \& Hudson, H. S. 1974, SoPh, 39, 155

Delaboudinière, J.-P., Artzner, G. E., Brunaud, J., et al. 1995, SoPh, 162, 291

De Pontieu, B., Title, A. M., Lemen, J. R., et al. 2014, SoPh, 289, 2733

Dennis, B. R. 1985, SoPh, 100, 465

Fletcher, L., Dennis, B. R., Hudson, H. S., et al. 2011, SSRv, 159, 19

Fludra, A., Griffin, D., Caldwell, M., et al. 2013, Proc. SPIE, 8862, 88620

Galsgaard, K., \& Nordlund, Å. 1996, JGR, 101, 13445

Goossens, M., Van Doorsselaere, T., Soler, R., \& Verth, G. 2013, ApJ, 768, 191

Gudiksen, B. V., Carlsson, M., Hansteen, V. H., et al. 2011, A\&A, 531, A154

Gudiksen, B. V., \& Nordlund, Å. 2005, ApJ, 618, 1020

Guerreiro, N., Hansteen, V., \& De Pontieu, B. 2013, ApJ, 769, 47

Haberreiter, M. 2011, SoPh, 274, 473

Haberreiter, M., Delouille, V., Mampaey, B., et al. 2014, JSWSC, 4, A30

Haisch, B., Strong, K. T., \& Rodono, M. 1991, ARA\&A, 29, 275

Halain, J.-P., Rochus, P., Renotte, E., et al. 2014, Proc. SPIE, 9144, 8

Handy, B. N., Acton, L. W., Kankelborg, C. C., et al. 1999, SoPh, 187, 229

Hansteen, V. H., Carlsson, M., \& Gudiksen, B. 2007, in ASP Conf. Ser. 368, The Physics of Chromospheric Plasmas, ed. P. Heinzel, I. Dorotovič \& R. J. Rutten (San Francisco, CA: ASP), 107

Hansteen, V. H., \& Gudiksen, B. 2005, in ESA Special Publication 592, Solar Wind 11/SOHO 16, Connecting Sun and Heliosphere, ed. B. Fleck, T. H. Zurbuchen \& H. Lacoste (Noordwijk: ESA), 483

Hansteen, V. H., Guerreiro, N., De Pontieu, B., \& Carlsson, M. 2015, ApJ, 811,106

Hansteen, V. H., Hara, H., De Pontieu, B., \& Carlsson, M. 2010, ApJ, 718, 1070

Hayek, W., Asplund, M., Carlsson, M., et al. 2010, A\&A, 517, A49

Hendrix, D. L., van Hoven, G., Mikic, Z., \& Schnack, D. D. 1996, ApJ, 470,1192
Hudson, H. S. 1991, SoPh, 133, 357

Klimchuk, J. A. 2006, SoPh, 234, 41

Klimchuk, J. A. 2015, RSPTA, 373, 40256

Krucker, S., \& Benz, A. O. 1998, ApJL, 501, L213

Lemen, J. R., Title, A. M., Akin, D. J., et al. 2012, SoPh, 275, 17

Lin, R. P., Schwartz, R. A., Kane, S. R., Pelling, R. M., \& Hurley, K. C. 1984, ApJ, 283, 421

Lu, E. T., \& Hamilton, R. J. 1991, ApJL, 380, L89

Maehara, H., Shibayama, T., Notsu, S., et al. 2012, Natur, 485, 478

Manno, I. 1999, Introduction to the Monte-Carlo Method (Budapest Akadémiai Kiadó)

Parker, E. N. 1987, SoPh, 111, 297

Parnell, C. E., \& De Moortel, I. 2012, RSPTA, 370, 3217

Parnell, C. E., \& Jupp, P. E. 2000, ApJ, 529, 554

Pesnell, W. D., Thompson, B. J., \& Chamberlin, P. C. 2012, SoPh, 275, 3

Peter, H., Gudiksen, B. V., \& Nordlund, Å. 2006, ApJ, 638, 1086

Reale, F. 2010, LRSP, 7, 5

Schaefer, B. E. 1989, ApJ, 337, 927

Schaefer, B. E., King, J. R., \& Deliyannis, C. P. 2000, ApJ, 529, 1026

Schrijver, C. J., Beer, J., Baltensperger, U., et al. 2012, JGRA, 117, 8103

Testa, P., De Pontieu, B., Allred, J., et al. 2014, Sci, 346, B315

Tomczyk, S., McIntosh, S. W., Keil, S. L., et al. 2007, Sci, 317, 1192

van Ballegooijen, A. A., Asgari-Targhi, M., Cranmer, S. R., \& DeLuca, E. E. 2011, ApJ, 736, 3

van Doorsselaere, T., De Groof, A., Zender, J., Berghmans, D., \& Goossens, M. 2011, ApJ, 740, 90

Walsh, R. W., \& Ireland, J. 2003, A\&AR, 12, 1

Withbroe, G. L., \& Noyes, R. W. 1977, ARA\&A, 15, 363

Yashiro, S., Akiyama, S., Gopalswamy, N., \& Howard, R. A. 2006, ApJL, $650, \mathrm{~L} 143$ 